\title{
Le droit au suicide médicalement assisté
}

\section{Regard sur le droit suisse, du Benelux et de la Cour EDH}

Cette contribution illustre le cadre légal suisse de l'aide médicale au suicide et des conditions d'octroi de Natrium Pentobarbital (NaP) notamment au regard du CP, de la LStup, de la LPTh et de la LPMéd, ainsi que le cadre réglementaire suisse actuel. Elle se penche aussi sur les traits saillants des systèmes du Benelux en matière de suicide assisté et de soins palliatifs dans le but d'examiner le potentiel de développement du système actuel en Suisse.

I. Introduction

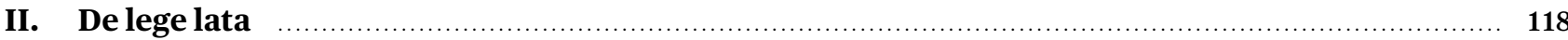

1. Position de la Cour EDH - Marge d’appréciation des État membres $\ldots \ldots \ldots \ldots \ldots \ldots \ldots \ldots \ldots \ldots \ldots \ldots \ldots$

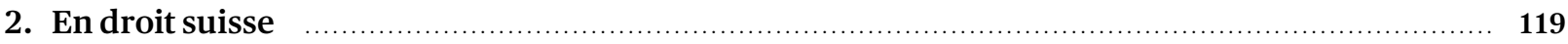

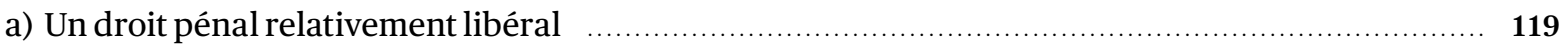

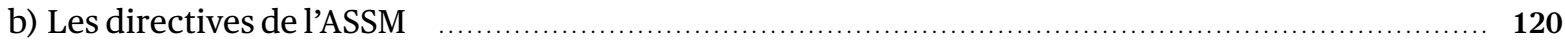

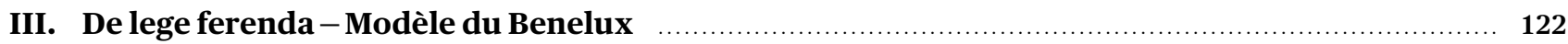

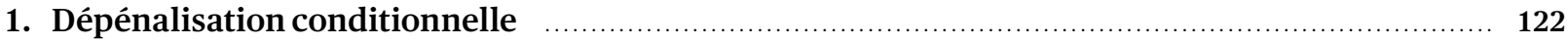

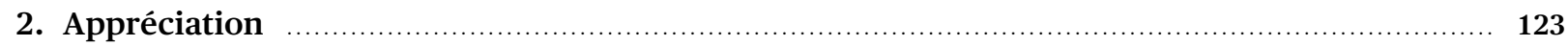

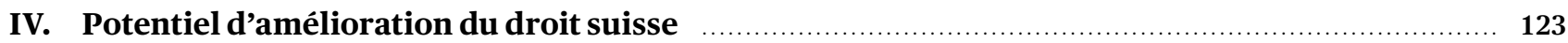

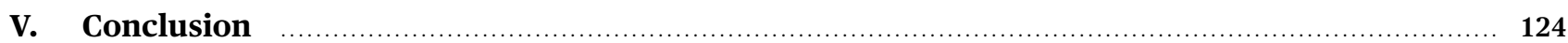

Citation:

FABIAN TEICHMANN / MADELEINE CAMPRUBI /

LÉONARD GERBER, Le droit au suicide médicalement

assisté, sui generis 2021, p. 117

Fabian Teichmann, Dr. iur. Dr. rer. pol., LL.M., Rechtsanwalt, Teichmann International (Schweiz) AG (teichmann@teichmann-law.ch); Madeleine Camprubi, PD Dr. iur., Rechtsanwältin, Teichmann International (Schweiz) AG (camprubi@teichmann-law.ch); Leonard Gerber, BLaw, Teichmann International (Schweiz) AG (gerber@teichmann-law.ch).

URL: sui-generis.ch $/ 175$

DOI: https://doi.org/10.21257/sg.175

Ce(tte) œuvre est mise à disposition selon les termes de la Licence Creative Commons Attribution - Partage dans les Mêmes Conditions 4.0 International. 


\section{Introduction}

1 «Tu ne tueras point». Cet adage tirant ses racines du Décalogue constitue le fondement de la société moderne et rayonne tant dans la mission centrale des professions médicales que dans le système juridique européen. À ce titre, la déclaration de Genève enjoint aux médecins de promettre par serment qu'ils veilleront au respect absolu de la vie humaine. ${ }^{1}$ En droit suisse, le meurtre est la première infraction réprimée par le Code pénal. Cependant, les affaires récentes traitées par la Cour européenne des droits de l'homme (Cour EDH) tendent vers la reconnaissance du droit à l'assistance médicale au suicide. Cette tendance à première vue diamétralement opposée au droit à la vie, garanti par la $\mathrm{CEDH}$, centre le débat vers la question du droit individuel à l'autodétermination et du droit de mourir dans la dignité.

2 Les tiers fournissant une assistance au suicide aux personnes désirant mettre fin à leurs jours risquent encore une répression pénale, si bien qu'il se pose la question de la place du droit à l'autodétermination des personnes désirant mettre fin à leurs jours et du rôle des médecins à cet égard. Cette contribution décrit la position de la Cour EDH ainsi que l'approche du droit suisse et du droit des pays du Benelux tendant vers une libéralisation de l'assistance médicale au suicide. Pour ce qui est de la Suisse, il sera traité de son droit pénal relativement libéral puis de son droit de la santé qui se caractérise par une réglementation pratique importante de la part de l'Académie suisse des sciences Médicales (ASSM). Nous décrirons ensuite l'approche singulière adoptée par le Benelux tendant à un contrôle a posteriori de la régularité de l'euthanasie exercée par le médecin et à l'immunité pénale du médecin pratiquant l'euthanasie. Nous tenterons de dresser les traits saillants de chacun de ces systèmes juridiques dans le but de déterminer des principes généraux et si une évolution des conceptions juridiques modernes se présente.

\section{De lege lata}

\section{Position de la Cour EDH - Marge d'appréciation des État membres}

3 Dans l'arrêt Alda Gross c. Suisse, la Cour EDH statuait pour la première fois en 2013 sur une demande d'assistance au suicide d'une demanderesse souhaitant mettre fin à ses jours sans justifier d'aucune maladie en phase terminale. ${ }^{2}$

1 URBAN WIESING / RAMIN PARSA-PARSI, L’Association médicale mondiale a révisé la Déclaration de Genève, Bulletin des médecins suisses 2018, vol.99, n. 8, p. 247 s.

2 Jugement du Cour EDH n67810/10 du 14 mai 2013 (Gross c. Suisse), \$21. Voir également DANIEL HÜRLIMANN, Kommentar zum jüngsten Suizidhilfe-Entscheid des Europäischen Gerichtshofs für Menschenrechte, Bulletin des médecins suisses, 2013; 94: 31/32, p. 1173.
Cet arrêt confirme une nouvelle tendance de la Cour EDH d'examiner les requêtes d'assistance au suicide non plus sur la base du droit à la vie, mais sur la base du droit au respect de la vie privée et familiale. ${ }^{3}$ En effet, pour la Cour EDH, «l'art. 2 CEDH ne saurait être interprété comme conférant un droit diamétralement opposé, à savoir un droit à mourir, il ne saurait davantage créer un droit à l'autodétermination en ce sens qu'il donnerait à tout individu le droit de choisir la mort plutôt que la vie».4 Il n'y a donc pas d'obligation positive des états parties de garantir aux justiciables un droit à mourir par des mesures législatives. ${ }^{5}$ S'agissant d'une prétention à une aide au suicide basée sur l'art. 8 CEDH, dans l'arrêt Haas c. Suisse la Cour avait toutefois reconnu en 2011 un droit de décider de quelle manière et à quel moment sa vie doit prendre fin, à condition que le justiciable soit en mesure de former librement sa volonté à ce propos et d'agir en conséquence. ${ }^{6}$ Cette position est confirmée dans l'arrêt Gross c. Suisse dans lequel la Cour statue qu'il incombe aux autorités internes des États membres d'émettre des orientations complètes et claires sur la question de savoir si et dans quelles circonstances une personne dans la situation de la requérante devrait se voir octroyer le droit d'acquérir une dose létale de médicaments lui permettant de mettre fin à ses jours. ${ }^{7}$ Par conséquent, la Cour préfère renvoyer à la marge d'appréciation des États parties et à leur législation interne sans toutefois se prononcer sur l'illicéité de l'euthanasie ou de l'assistance au suicide par rapport à la Convention. ${ }^{8}$ Le droit à la protection de la vie privée et familiale consacre ainsi un droit au choix de mourir, mais non un droit d'exiger l'aide de l'État pour mettre fin à ses jours. ${ }^{9}$ Chaque individu peut décider de la manière de mettre fin à ses jours sur la base de l'art. 8 $\mathrm{CEDH}$, ce dernier article n'emportant toutefois aucune obligation positive de l'État de fournir une assistance au suicide en distribuant une substance létale, d'autre part sans ordonnance médicale. ${ }^{10}$

3 HECTOR ENTENZA, La réglementation légale suisse en matière d'accès à l'assistance au suicide: Réflexions autour de l'arrêt Gross c. Suisse, RSDIE 2014, p.190; Voir également Jugement du Cour EDH $\mathrm{n}^{\circ} 67810 / 10$ du 14 mai 2013 (Gross c. Suisse), §60.

4 Jugement Cour EDH n ${ }^{\circ}$ 2346/02 du 29 avril 2002 (Pretty c. RoyaumeUni), §39.

5 ENTENZA (n.3), p. 192.

6 Jugement Cour EDH n ${ }^{\circ} 31322 / 0720$ janvier 2011 (Haas c. Suisse), §51, puis confirmé dans le Jugement du Cour EDH n67810/10 du 14 mai 2013 (Gross c. Suisse), \$21.

7 Jugement du Cour EDHn ${ }^{\circ} 67810 / 10$ du 14 mai 2013 (Gross c. Suisse), \$69.

8 ENTENZA (n.3), p.191 s., avec références.

9 ENTENZA (n.3), p.193.

10 Plus concrètement voir Jugement Cour EDH n ${ }^{\circ} 31322 / 07$ 20janvier 2011 (Haas c. Suisse), \$61 indiquant que «Compte tenu de ce qui précède, et eu égard à la marge d'appréciation dont disposent dans ce domaines les autorités internes, la Cour estime que, même à supposer que les Etats aient une obligation positive d'adopter des mesures permettant de faciliter la commission d'un suicide dans la dignité, les autorités suisses n'ont pas méconnu cette obligation en l'espèce», voir également ENTENZA (n.3), p.193, ainsi que HÜRLIMANN, Kommentar (n. 2), p.1174. 


\section{En droit suisse}

a) Un droit pénal relativement libéral

aa) Pénalisation de l'assistance au suicide en cas de mobile égoïste

4 Le Code pénal Suisse réprime les infractions contre la vie et l'intégrité corporelle aux art.111-136 CP-CH. Plus particulièrement, l'art. $115 \mathrm{CP}-\mathrm{CH}$ érige l'incitation et l'assistance au suicide au rang d'infraction en la réprimant avec jusqu'à 5 ans de peine privative de liberté ou avec une peine pécuniaire. Par principe, le tiers apportant son aide au suicide de la victime n'est pas sanctionné, ceci à moins qu'il n'ait été «poussé par un mobile égoïste», sans que la loi définisse plus précisément cette dernière notion.

5 Par «mobile égoïste», la pratique comprend que l'auteur doit tendre principalement à satisfaire ses intérêts personnels, peu importe que ceux-ci soient d'ordre matériel ou affectif. ${ }^{11}$ La pratique consacre en outre le critère de la maîtrise effective (Tatherrschaft) de l'acte mortifère, à savoir si la personne concernée a elle-même mis fin à sa vie ou si un tiers la lui a enlevée. ${ }^{12}$ Selon la doctrine et la jurisprudence, parallèlement à la maîtrise effective de l'acte mortifère, la victime doit aussi prendre la décision de se suicider sur la base d'une responsabilité personnelle, la capacité de discernement doit alors être donnée (au sens de l'art.16 du CC). ${ }^{13}$ La capacité de discernement chez les personnes majeures est présumée. ${ }^{14}$ Le fardeau de la-difficile-preuve de l'incapacité de discernement au moment de la mise à exécution du suicide incombe à l'autorité d'accusation. Cela devrait expliquer la rareté des condamnations sur la base de l'art. $115 \mathrm{CP}-\mathrm{CH} .{ }^{15}$

6 Toutefois, les organisations d'aide au suicide risquent une procédure pénale, en raison de la formulation abstraite de la notion de «mobile égoïste» et plus exactement, du fait que le cadre de la rémunération admissible de l'organisation d'aide au suicide demeure peu claire en

11 MADELEINE HIRSIG-VOUILLOZ, La responsabilité du médecin, Genève 2017, p. 165.

12 CHRISTIAN SCHWARZENEGGER / MAKOTO IDA, Autonomie am Lebensende - Kultur und Recht Die aktuelle Diskussion in der Schweiz und in Japan, Zurich 2018, p.140.

13 FRANZ WERRO / IRÈNE SCHMIDLIN, dans: Pichonnaz/Foëx (éd.), Commentaire Romand, Code Civil I (CC), Bâle 2010, art.16 N 4.

14 Voir l'ATF 108 V 121, p. 126 c. 4 ainsi que l'ATF 90 II 9, p. 12 c. 3. Voir également ROLAND FANKHAUSER, dans: Geiser/Fountoulakis (Hrsg.), Basler Kommentar zum Zivilgesetzbuch I (Art. 1-456 ZGB), 6. éd., Bâle 2018, art.16 N 47 .

15 Voir à cet égard, MARKUS ZIMMERMANN / STEFAN FELDER / URSULA STRECKEISEN / BRIGITTE TAG, Das Lebensende in der Schweiz - Individuelle und gesellschaftliche perspektiven, Bâle 2019, p.135ss., CHRISTIAN SCHWARZENEGGER, dans: Niggli/Wiprächtiger (Éd.), Basler Kommentar, Strafrecht, 4. éd., Bâle 2019, art.115 (cit. BSK StGB-ÉDITEUR) raison d'un manque de précédents judiciaires. ${ }^{16}$ La réception d'un paiement d'une cotisation de membre, pris en compte en tant que coût administratif, peut constituer un soupçon préalable d'une violation de l'art. 115 CP-CH. ${ }^{17}$ En 2014, l'Office fédéral de la statistique a enregistré 742 suicides assistés, correspondant à 1.4\% des décès en Suisse. ${ }^{18}$ Elle révèle ainsi une augmentation de $26 \%$ en un an et que cette tendance ne cesse d'augmenter depuis 2008.19 Or, il convient de mentionner une affaire pénale zurichoise dans laquelle le tribunal de district libéra le fondateur de Dignitas des chefs d'accusation d'assistance au suicide (art.115 CP) et usure (art.157 CP) répétées le 1 juin $2018 .^{20}$ Le tribunal s'est basé sur la position d'Häring en jugeant le modèle d'affaire de ladite organisation d'aide au suicide et a considéré que le ministère public n’a pas prouvé que le prévenu ait agi de manière «prédominament» égoïste, malgré le système de rémunération. ${ }^{21}$ Le jugement de ce Tribunal d'arrondissement est entré en force, à défaut d'appel transmis par le ministère public auprès du tribunal cantonal de Zurich en temps nécessaire. ${ }^{22}$

L'art.115 CP-CH est exhaustif quant à l'assistance d'un 7 tiers au suicide. ${ }^{23}$ Cette dernière englobe également la mise à disposition de moyens létaux ou d'une prescription dans un but de suicide, pour autant que le tiers n'ait pas accompli l'acte mortifère et qu'il soit motivé par un mobile égoïste. ${ }^{24}$ Toute autre mise à exécution de la mort de la part d'un tiers constitue un homicide sous forme de meurtre (art.111 CP-CH), d'assassinat (art.112 CP-CH), de meurtre passionnel (art.113 $\mathrm{CP}-\mathrm{CH}$ ) ou de meurtre sur

16 Voir à cet égard ZIMMERMANN/FELDER/STRECKEISEN/TAG (n.15), p. 91. BSK StGB-SCHWARZENEGGER, Art. 115 N14 avec les références citées.

17 Voir à cet égard l'arrêt du Tribunal fédéral 1B_516/2011 du 17 novembre 2011 c. 2.4 où le Tribunal fédéral concluait qu'il n'y avait pas de violation des droits de la personnalité de l'association d'aide au suicide.

18 Voir Actualité OFS 2014 datée d'octobre 2016 «Statistique des causes de décès 2014 - Suicide assisté et suicide en Suisse », p.1.

19 Actualité OFS 2014 datée d'octobre 2016 «Statistique des causes de décès 2014-Suicide assisté et suicide en Suisse», p.1. Le rapport est de 10 hommes pour 13 femmes recourant à l'aide au suicide.

20 Arrêt du Tribunal de district de Uster du GG170037-I/Mc/UO2/gp du 31 mai 2018 VIII-X c. 1 ss. Pour une critique de l'arrêt, voir DANIEL HÜRLIMANN, Suizidhilfe ohne Kenntnis des Gesundheitszustands, HSG Focus 3/2019: «Gesundheit»-Dossier; Voir également, RETO FLURY, Der Staatsanwaltschaft kritisiert das Minelli-Urteil als zu einseitig, NZZ du 2 juin 2018.

21 Selon le tribunal, «für eine Verurteilung müssen gemäss juristischer Literatur überwiegend selbstsüchtige Beweggründe vorliegen». Voir à cet égard, DANIEL HÄRING, Fünf Mythen über Suizidhilfeorganisationen, Jusletter 8 mai $2017, \mathrm{~N}^{\circ} 56$ et 71 .

22 NZZ du 20 août 2020 (Freispruch für Dignitas-Gründer Ludwig A. Minelli wird rechtskräftig).

23 BSK StGB-SCHWARZENEGGER, Art. 115 N 2 avec références.

24 Voir notamment THOMAS GÄCHTER / THUY XUAN TRUONG, Die Rolle der Ärzteschaft in der Sterbehilfe, insbesondere bei der Verschreibung von Natriumpentobarbitural, Praxis 2019/108, p. 194. 
demande de la victime (art.114 $\mathrm{CP}-\mathrm{CH}$ ). Cette dernière forme d'homicide est punie moins sévèrement, à savoir de jusqu'à 3 ans de PPL ou d'une PPEC, elle intervient notamment lorsque l'auteur « cède à un mobile honorable» comme la «pitié» en tuant «sur demande sérieuse et instante» de la victime.

\section{bb) La loi fédérale sur les stupéfiants et la loi fédérale sur produits thérapeutiques - le NaP}

8 Les personnes sollicitant une aide au suicide en Suisse recourent en premier lieu au Natrium Pentobarbital qui a pour effet d'endormir paisiblement le patient avant d'entraîner sa mort. ${ }^{25}$ Ce psychotrope ${ }^{26}$ est soumis à la loi sur les stupéfiants ainsi qu'à loi fédérale sur les produits thérapeutiques lorsqu'il est utilisé comme produit thérapeutique, à savoir comme médicament ou dispositif médical. ${ }^{27}$ Seuls les médecins (et les médecins-vétérinaires) exerçant leur profession sous leur propre responsabilité professionnelle sont en mesure de prescrire du NaP par formule d'ordonnance ordinaire et selon les règles reconnues des sciences pharmaceutiques et médicales, ainsi que dans la mesure admise par la science. ${ }^{28}$ À notre sens, un devoir de notification aux autorités cantonales compétentes s’impose sous peine de sanctions pénales. ${ }^{29}$

25 Le NaP correspond à un sel de pentobarbital figurant au tableau b des substances soumises à contrôle du Département fédéral de l'intérieur. Voir à cet égard les art. 1 al. 2 let. a et. b ainsi que l'Annexe 3 de l'Ordonnance du DFI sur les tableaux des stupéfiants, des substances psychotropes, des précurseurs et des adjuvants chimiques du 30 mai 2011(OTStup-DFI; RS 812.121.11), ainsi que l'art. 3 al. 2 let. b de l'Ordonnance sur le contrôle des stupéfiants du 25 mai 2011 (OCStup; RS 812.121.1),

26 Voir Tableau III de la Convention sur les substances psychotropes du 21 février 1971 (RS 0.812.121.02) ainsi que GÄCHTER/TRUONG (n. 24), p. 194

27 Loi fédérale sur les médicaments et les dispositifs médicaux du 15 décembre 2000 (LPTh ; RS 812.21); Loi fédérale sur les stupéfiants et les substances psychotropes du 3 octobre 1951 (LStup; RS 812.121). Voir les art. 2 al. 1 let. a et b LPTh, l'art. $1 b$ LStup ainsi que l'Annexe 3 OTStup-DFI. Conformément à l'art. 2 LStup, «la LStup est applicable lorsque la LPTh ne prévoit pas de réglementation ou que sa réglementation est moins étendue». Néanmoins, à l'heure actuelle, aucun médicament à usage humain renfermant du pentobarbital n'est autorisé par Swissmedic, alors qu'au contraire cinq médicaments vétérinaires renfermant du pentobarbital ont été autorisés, voir à cet égard listes de medicamentes; Voir également GÄCHTER/TRUONG (n.24), p.194.

28 Conformément aux art. 26 LPTh, aux art.10 et 11 LStup et à l'art. 46 al. 3 OCStup. Voir également l'arrêt du Tribunal régional du Littoral et du Val-de-Travers POL.2011.256 (EXT.2013.10) du 8 juillet 2013, qui «Reconnaît X. coupable d'infraction à l'art. 26 LPTh» résumé dans HÜRLIMANN, Kommentar (n. 2), p.1173, n.b.p. 19. Selon les c. I/d et III/a, les directives de l'ASSM ne constituent pas des règles de droit mais permettent d'interpréter les règles légales, spécialement lorsqu'il s'agit de mesure la diligence d'une personne astreinte à une déontologie professionnelle dans le domaine médical notamment en lien avec l'art. 26 LPTh.

29 Voir les art.11 al. $1^{\text {bis }}$ et 21 LStup. Un tel devoir correspondrait à l'esprit de l'art. 11 al. $1^{\text {bis }}$ LStup car les travaux parlementaires (Rapport de la Commission de la sécurité sociale et de la santé publique du Conseil national sur l'initiative parlementaire, Révision partielle de la loi sur les stupéfiants [FF 2006 8141], p. 8176) indique qu'une telle norme vise à «contrôler la prescription par les professionnels de la
La LStup et la LPTh ne concrétisent toutefois ni un droit au suicide ni la procédure et les conditions nécessaires à l'obtention de NaP.

Le Tribunal fédéral reconnaît dans l'ATF 133 I 58 bien 9 plus un droit au suicide avec un rang de droit constitutionnel individuel et de droits de l'homme au même titre que le droit à la vie. ${ }^{30}$ Il statue qu'il n'est pas anticonstitutionnel d'exiger une ordonnance médicale pour la remise de NaP. ${ }^{31}$ Cette obligation d'ordonnance pour la fourniture de NaP ne viole pas le droit fondamental à une aide au suicide. ${ }^{32}$ Le Tribunal fédéral se réserve toutefois de fixer les conditions et la procédure requise pour l'octroi de NaP. Néanmoins, pour déterminer la conformité au droit de l'octroi de l'aide au suicide par un médecin à un patient, il se réfère aux règles professionnelles des médecins ou des directives de l'Académie Suisse des Sciences médicales (ci-après ASSM). ${ }^{33}$ Il renvoie en particulier aux conditions des directives de l'ASSM du 25 novembre 2004 "Prise en charge des patientes et patients en fin de vie », ${ }^{34}$ dont il sera question au prochain chapitre, renvoyant elles-mêmes au Code de déontologie de la Foederatio Medicorum Helveticorum (FMH) ${ }^{35}$ à ses art.17 et $18 .{ }^{36}$

\section{b) Les directives de l'ASSM}

Les directives de l'ASSM dans leur version du 25 novembre 10 $2004^{37}$ rappellent qu'il n'est pas du devoir du médecin de proposer une aide au suicide, il a au contraire «le devoir de soulager les souffrances qui pourraient être à l'origine d'un désir de suicide ».38 Cependant, le désir du patient de mourir est reconnu par l'ASSM, et la décision morale personnelle du médecin d'apporter une aide au suicide d'autre part doit être respectée. ${ }^{39}$ Il incombe toutefois au médecin de vérifier préalablement que la fin de vie soit

santé dans les domaines où les règles médicales font encore défaut afin d'éviter les abus ». Toutefois, l'association des pharmaciens cantonaux dans la version alémanique de son interprétation technique datée du 25 octobre 2017 indique qu'une copie de l'ordonnance «peut» être adressée au médecin cantonal (Kannvorschrift).

30 ATF 133 I 58 c. 6.2.1.

31 ATF 133 I 58 c. 6.3.2 et 6.3.6.

32 En vertu de l'art. 11 LStup, les professions médicales sont toutefois tenues den'en prescrire que dans une mesure admise par la science. Voir également l'ATF 133 I 58 c. 6.3.2. et 6.3.6.

33 ATF 133 I 58 c. 6.3.4. Voir néanmoins l'interprétation technique de l'association des pharmaciens cantonaux du 25 octobre 2017.

34 Directives de l'ASSM.

35 Code de déontologie de la Foederatio Medicorum Helveticorum (FMH); ci-apres FMH.

36 Voir l'art.18 FMH.

37 Les directives de l'ASSM «Prise en charge des patientes et patients en fin de vie »; cette version a été adaptée au $1^{\mathrm{er}}$ janvier 2013 au droit de la protection des adultes.

38 Directives de l'ASSM, p.9; Voir également la prise de position de la CCE 2012, point 2. Voir également ZIMMERMANN/FELDER/STRECKEISEN/TAG (n.15), p.17.

39 Voir également les directives de l'ASSM « La capacité de discernement dans la pratique médicale» du 29 novembre 2018, p.18. 
proche, que le patient soit capable de discernement, ${ }^{40}$ que son désir de mourir soit mûrement réfléchi sans pression extérieure et persistant et enfin que des alternatives de traitement aient été proposées et qu'elles soient mises en œuvre si souhaitées par le patient.41

La maladie dont souffre le patient doit rendre une mort prochaine inéluctable. ${ }^{42}$ Le médecin doit avoir acquis en se fondant sur « des signes cliniques» «la conviction qu'un processus s'est installé dont on sait par expérience qu'il entraîne la mort en l'espace de quelques jours ou de quelques semaines ". ${ }^{43}$ Avec leurs directives du 17 mai 2018 «Attitude face à la fin de vie et à la mort», l'ASSM a étendu le champ d'application de l'aide médicale au suicide, sous certaines conditions, aux patients souhaitant une aide médicale pour mettre fin à leur vie que la mort soit imminente ou non. Ainsi, l'aide au suicide relève du domaine de compétence de la médecine en présence de symptômes ou de limitations fonctionnelles tangibles causant au patient « une souffrance insupportable». Cette version révisée n’a pas été ratifiée par la FMH qui objecte que ce dernier critère est indéfinissable. ${ }^{44}$ Cela n'empêche pas les tribunaux de se baser sur la version révisée dans le cadre de l'interprétation du devoir de soin et de conscience professionnelle de l'art. 40 lit. a LPMéd ${ }^{45}$ par les autorités d'application de la loi. ${ }^{46}$

40 Voir à cet égard l'arrêt du Tribunal cantonal de Bâle-Campagne 300 18245 cour pénale du 9juillet 2019 c. 1.2.6.h qui soulève l'importance d'un deuxième avis médical indépendant avant l'octroi d'une aide au suicide à une personne incapable de discernement. Dans le cas mentionné, la cour plénière a retenu de multiples violations aux règles professionnelles, notamment à la LPTh, de l'accusée ayant unilatéralement prodigué une aide au suicide à une patiente souffrant de troubles psychiques, sans avoir préalablement établi une expertise psychiatrique attestant de sa capacité de discernement, alors qu'elle ne disposait pas elle-même d'une formation en psychiatrie. La présidente de l'association d'aide au suicide a été libérée du grief de meurtre intentionnel et a été condamnée à une PPL de 15 mois avec sursis ainsi que d'une amende de 20 '0oo CHF ainsi que d'une interdiction de prescrire une aide au suicide à des patients atteints de trouble psychiques pour violation de la LPTh Voir également l'arrêt du Tribunal fédéral 2C_410/2014 du 22 janvier 2015 c. 7 , ainsi que l'arrêt du Tribunal fédéral 2C_9/2010 du 10 avril 2010 c. 3.2 (ungenügende Prüfung der Urteilsfähigkeit).

41 Directives de l'ASSM, p. 9 (il incombe au médecin «la responsabilité de vérifier si les exigences minimales suivantes sont réunies », etc.). Voir également la prise de position de la CCE 2012, point 2.

42 Directives de l'ASSM, p. 9, «La maladie dont souffre le patient permet de considérer que la fin de la vie est proche».

43 Voir à cet égard, la prise de position de la Commission Centrale d'Éthique datée du 20 janvier 2012.

44 GÄCHTER/TRUONG (n.24), p. 195 ainsi que la note de bas de page n ${ }^{\circ} 5$ avec références.

45 Loi fédérale sur les professions médicales universitaires du 23 juin 2006 (LPMéd; RS 811.11).

46 Voir les positions semblables de GÄCHTER/TRUONG (n.24), p.195 et de LAURA PULTRONE, Rezeptierung von NaP für die Suizidbeihilfe, Unter besonderer Berücksichtigung der Möglichkeit einer Rezeptur an gesunde Personen, dans: Coninx/Ege/Mausbach (éd.), Prävention und freiheitliche Rechtsordnung, Zürich 2019, p.188ss.; ainsi que FABIAN TEICHMANN / MADELEINE CAMPRUBI, Baustelle
La question se complique s'agissant de l'évaluation de la 12 capacité de discernement des personnes atteintes de démence. ${ }^{47}$ Le médecin doit se baser sur la volonté présumée du patient, en se référant le cas échéant aux directives anticipées qu’il a rédigées, au représentant thérapeutique nommé par procuration ou s'il a clairement exprimé sa volonté à ses proches. ${ }^{48}$ D'autre part, le médecin doit s'assurer du désir persistant du patient de se donner la mort. Il doit avoir mené plusieurs entretiens, dans lesquels le patient a maintes fois réitéré son désir persistant de se donner la mort, malgré que des alternatives lui aient été proposées par le médecin. ${ }^{49}$

En cas de violation de ces dispositions, les médecins sont 13 soumis à des sanctions disciplinaires. ${ }^{50}$ La compétence demeure auprès des cantons pouvant infliger une interdiction du médecin de pratiquer sous sa propre responsabilité, temporairement jusqu'à 6 ans ou voire définitivement. ${ }^{51}$ Le FMH prévoit à son art. 47 des sanctions similaires en cas de violation des devoirs dans l'exercice de la profession médicale. 52

Concrètement, la loi ne prévoit pas de droit général à 14 obtenir du NaP. En vertu de l'obligation d'ordonnance, cette substance létale est réservée aux personnes disposant d'une prescription médicale. Depuis la révision des directives de l'ASSM, toutefois non ratifiée par la FMH, non seulement les patients dont la mort est imminente, mais aussi ceux dont les souffrances sont insupportables entrent en compte pour l'octroi de NaP.53 Il existe donc un vide juridique en dehors du cadre réglementaire fixé

Suizidhilfe: Herausforderungen für den Gesetzgeber und die Justiz, Pflegerecht 2020, p. 28. Selon PULTRONE, les règlements privés ne sont pas contraignants, mais peuvent servir de fil rouge. La question de savoir si la version précédente des directives de l'ASSM remplacée lors de sa révision, fait encore partie du FMH peut donc rester ouverte.

47 Voir les directives de l'ASSM « Prise en charge et traitement des personnes atteintes de démence » du 16 novembre 2017, p.10, ainsi que EVA MARIA BELSER / SANDRA EGLI, Das Recht auf einen selbstbestimmten Tod, ZBJV 156/2020, p. 407ss.

48 Directives de l'ASSM « Prise en charge et traitement des personnes atteintes de démence $»$, p. 3 .

49 ATF 133 I 58 c. 6.3.4.

50 Voir l'art. 43 al. 1 LPMéd.

51 Voir l'art. 34 LPMéd.

52 Celles-ci comprennent une amende jusqu'à 50’000 CHF, la suspension temporaire de la qualité de membre ou exclusion de la FMH et de la société cantonale de médecine (art. 47 FMH). L’autorisation extra-cantonale limitée à 90 jours de l'art. 35 al. 2 LPMéd devrait également être rejetée en cas de violation du devoir de diligence médical (voir également le jugement du Tribunal d'appel de Bâle-Ville du 6 juillet 2017, selon lequel « die Vorinstanz das Bewilligungsgesuch zu Unrecht nur wegen ärztlicher Suizidhilfe abgewiesen habe».

53 Ànotre sens, c'est doncà juste titre que BELSER/EGLI(n. 47) indiquent que les personnes désirant se procurer du NaP sans être en fin de vie devraient consulter un médecin non-affilié à la FMH et ne risquant pas de sanctions disciplinaires de la FMH, voir à cet égard BELSER/ EGLI (n.47), p. 395 et les références citées. 
par les directives de l'ASSM, les médecins étant soumis à des risques et des sanctions disciplinaires lourdes s'ils ne s'y tiennent pas pour octroyer du NaP. Ainsi que mentionné plus haut, pour le Tribunal fédéral, cette situation juridique ne viole pas le droit fondamental de mettre fin à sa vie. L'état ne serait pas tenu à garantir que les personnes désirant se suicider puissent obtenir du NaP, que ce fût avec ou sans ordonnance. ${ }^{54}$ Cet arrêt de principe ne traite toutefois que de la distribution de NaP. ${ }^{55}$ Que les alternatives au NaP soient plus douloureuses ou sujettes à plus de risques, n’y change rien aux yeux du Tribunal fédéral.56 Il convient de rappeler que le Tribunal fédéral s'est retenu de fixer les conditions et la procédure nécessaires à l'octroi du NaP.

\section{De lege ferenda - Modèle du Benelux}

15 Les pays du Benelux ont adopté une approche similaire à l'assistance médicale au suicide qui différente quelque peu de celle de la Suisse. ${ }^{57}$ Un aperçu de cette approche nous permettra d'en analyser les traits saillants ainsi que de déterminer l'évolution législative à l'exemple des juridictions plus libérales quant à l'assistance médicale au suicide faisant écho également à la jurisprudence de la Cour EDH.

\section{Dépénalisation conditionnelle} l'euthanasie et l'assistance au suicide sont progressivement entrées en vigueur consacrant une exceptio medica dépénalisant ${ }^{58}$ l'euthanasie médicalement assistée sous respect de critères de diligence. ${ }^{59}$ Une Commission indépendante se charge de contrôler à posteriori le respect

54 ATF 133 I 58 c. 6.3.6. Voir également l'arrêt du Tribunal fédéral 2C_9/2010 du 12 avril 2010 c. 2.4. et 3.2.

55 À cet égard GÄCHTER/TRUONG (n.24), p.196 à la note de bas de page $\mathrm{n}^{\circ} 18$.

56 ATF 133 I 58 c. 6.2.3. Concernant les alternatives voir par exemple KALLIA GAVELA, Ärztlich assistierter Suizid und organisierte Sterbehilfe, thèse Heidelberg 2013, p. 78.

57 Ces questions concernent également le Tribunal fédéral consitutionnel de l'Allemagne, voir par exemple «Verbot der geschäftsmäs sigen Förderung der Selbsttötung verfassungswidrig», ainsi que la Cour constitutionnelle de l'Autriche, voir par exemple «Es ist verfassungswidrig, jede Art der Hilfe zur Selbsttötung ausnahmslos zu verbieten ».

58 Voir à cet égard ZIMMERMANN/FELDER/STRECKEISEN/TAG (n.15), p.16.

59 Loi belge relative à l'euthanasie $\mathrm{N}^{\circ} 2002009590$ du 28 mai 2002, p. 28525; Wet toetsinglevensbeëindiging op verzoek en hulp bij zelfdoding, du 12 avril 2001 (Loi néerlandaise sur le «contrôle de l'interruption de la vie sur demande et de l'aide au suicide»); Loi Luxembourgeoise sur l'euthanasie et l'assistance au suicide JO Mémorial A46 de 2009 du 16 mars 2009; Voir également KENNETH CHAMBAERE et al., Recent trends in euthanasia and other end-of-life practices in Belgium, New England Journal of Medecine, vol. 372, N12, 2015, p.1179, des critères de diligence. ${ }^{60}$ Elles confèrent également aux patients le droit de refuser toute intervention diagnostique ou thérapeutique, ainsi que l'acharnement thérapeutique notamment par des dispositions de fins de vie ainsi que la désignation d'un représentant privé. ${ }^{61} \mathrm{Le}$ médecin peut ainsi reconstituer la volonté du patient en cas d'incapacité.

À titre d'exemple, les conditions de diligence suivantes 17 doivent être remplies pour que le médecin puisse prétendre à l'immunité pénale en Belgique. ${ }^{62}$ Le patient doit être majeur, capable et conscient au moment de sa demande (1), sa demande doit être formulée de manière volontaire, réfléchie et, le cas échéant, répétée et ne pas résulter d'une pression extérieure (2). Le patient doit se trouver dans une situation médicale sans issue et faire état d'une souffrance physique ou psychique constante et insupportable sans perspective d'amélioration (3). La demande du patient d'avoir recours à une euthanasie ou une assistance au suicide doit être consignée par écrit (4). Sur les conditions de formes, le médecin doit informer le patient, s'assurer de la persistance de la souffrance physique ou psychique et consulter un autre médecin indépendant (5). Enfin, il doit s'informer auprès de la Commission indépendante de l'existence de dispositions

BREGJE D. ONWUTEAKA-PHILIPSEN et al., Trends in End-of-life practices before and after the enactment of the euthanasia law in the Netherlands from 1990 to 2010 : a repeated cross-sectional survey, The Lancet, vol.380, 2012, p. 908 ss., OLIVIER GUILLOD, Soins et respect de la volonté du patient en fin de vie : regard de droit comparé, Jusletter 31 janvier 2011, N54, MYRIAM PIERRAT, Soins et respect de la volonté de la personne en fin de vie, Annales Droit Luxembourgeois 2009, p. 203, Rapport du DFJP daté du 15 mai 2009, Assistance organisée au suicide : Examen approfondi des solutions envisageables et de la nécessité d'une nouvelle règlementation fédérale (ci-après Rapport du DFJP), p. 18 s., ainsi que MICHEL PÂQUES, Avortement, euthanasie et Cour constitutionnelle, 2019, p.19.

60 GABRIELLE STEFFEN, Voltooid leven - une vie accomplie, Jusletter 27 août 2018, N35, AGNES VAN DER HEIDE, Physicians-Assisted Dying in the Netherlands, dans: Borasio/Jox/Taupitz/Wiesing (Éd.), Assistierter Suizid : der Stand der Wissenschaft, Berlin 2017, p. 21 ss. Voir également Rapport du DFJP (n. 59), p.18 s. Voir également NADÈGE BELDI, La problématique de l'assistance au suicide en droit Suisse, Jusletter du 20 octobre 2008, N50 ss., p. 2 s.

61 Voir art. 15 al. 2 de la loi luxembourgeoise du 16 mars 2009 sur l'euthanasie et l'assistance au suicide, voir également PIERRAT (n. 59), p. 190.

62 CP-BE art. 418 et 422 bis ainsi que l'art. 3 \$2 de la loi belge du 28 mai 2002 sur l'euthanasie; CP-PB art. 293 al. 2 et 294 al. 2; CP-LU art. 397-1 al.1; Voir également STEFFEN (n. 60), par. 31 et 36, AGNES VAN DER HEIDE / JOHANNES J. VAN DELDEN / BREGJE ONWUTEAKA-PHILIPSEN, End-of-Life Decisions in the Netherlands over 25 years, New England Journal Medicine, vol. 377, N 5, 2017, p. 492 s., JACQUES MASSION, Les lois belge et néerlandaise sur l'euthanasie, RGDM 2004, vol. 5, p. 211, BÉATRICE WELSCHINGER, La légalisation de l'euthanasie et de l'aide au suicide aux Pays-Bas: Un défi pour les États européens, InfoKara 2001/3, vol.16, p.3ss., JOHN GRIFFITHS, Les débats qui subsistent après l'encadrement légal de l'euthanasie aux Pays-Bas, Revue française des affaires sociales 2002, vol.3, p.173ss., ainsi que SUZANNE RAMEIX, Le Droit de Mourir, Gérontologie et société, n'108 mars 2004, vol. 27, p. 104 voir n.b.p. 3, ALAIN DE NAUW, Initiation au droit pénal spécial, Diegem 2008, 311, N 533 ; HERMAN NYS, La médecine et le droit, Diegem 1995, N723 s. 
de fin de vie au nom du patient. ${ }^{63}$ La loi n'impose toutefois pas la manière dont doit être posé l'acte qui met fin intentionnellement à la vie, elle s'applique donc également à l'assistance au suicide par remise de NaP. ${ }^{64} \mathrm{Le}$ médecin ayant pratiqué l'euthanasie doit soumettre un rapport à la Commission fédérale de contrôle, composé de médecins, praticiens et de psychiatres contrôlant $a$ posteriori la régularité de l'acte pratiqué. ${ }^{65}$ Si elle estime que les conditions n'ont pas été respectées, elle transmet sa décision motivée au médecin ainsi qu'au Collège médical disposant d'un délai d'un mois pour ouvrir une procédure disciplinaire. ${ }^{66}$ Elle peut également transmettre le dossier au parquet si elle estime que les conditions légales n'ont pas été respectées. Même si elle estime que les conditions légales ont été respectées, une action civile et/ou pénale reste ouverte dont le dernier mot reviendra au juge. ${ }^{67}$

\section{Appréciation}

18 Sur la base de ces développements, le Benelux a largement dépassé les autres pays européens en allant jusqu'à dépénaliser l'euthanasie directe par un médecin sous des conditions et une procédure stricte. ${ }^{68}$ Pour cela, une commission formée d'experts médicaux, juridiques et voire de psychiatres, doit observer a posteriori que les conditions posées par la loi et que les règles de déontologie médicale aient été respectées. Ces conditions sont fixées par une loi au sens stricte trahissant une tendance en Europe vers l'acceptation de l'assistance au suicide. En droit suisse, l'euthanasie directe est interdite, mais l'aide au suicide est toléré sous le joug de l'art. $115 \mathrm{CP}-\mathrm{CH}$ pour autant qu'aucun mobile égoïste ne soit imputable. L’ASSM, une entité privée suisse, tente de définir le cadre pratique de l'exercice des professions médicales et tend vers une lente libéralisation de l'assistance médicale au suicide. Il est toutefois douteux que, s'agissant de la remise de $\mathrm{NaP}$ par ordonnance, le manque de précision au niveau de la loi fédérale et le système réglementaire puissent résister à un futur examen de la Cour EDH. ${ }^{69}$

\footnotetext{
63 PIERRAT (n. 59), p. 212.

64 ETIENNE MONTERO, Suicide, euthanasie et suicide assisté-Le rôle de la loi face aux demandes de mort, dans journée d'étude sur le suicide, l'euthanasie et le suicide assisté, Bruxelles le 5 février 2009, p. 5.

65 Voir l'art. 8 de la loi belge du 28 mai 2002 sur l'euthanasie, voir également PÂQUES (n. 59), p. 19. STEFFEN (n. 60), N35, voir également MARTIN SCHUBARTH, Assistierter Suizid und Tötung auf Verlangen, ZStrR 2009, p.12s.

66 PIERRAT (n. 59), p. 216.

67 PIERRAT (n. 59), p. 216.

68 ZIMMERMANN/FELDER/STRECKEISEN/TAG (n.15), p.16.

69 Voir la critique adressée à la Suisse dans Jugement du Cour EDH $\mathrm{n}^{\circ} 67810 / 10$ du 14 mai 2013 (Gross c. Suisse), \$67. Pour une étude détaillée voir BELSER/EGLI (n. 47), p. 413 ss., ainsi que ENTENZA (n. 3), p. 190 .
}

\section{Potentiel d'amélioration du droit suisse}

Dans l'arrêt Gross c. Suisse, la Cour EDH a conclu à la vio- 19 lation du droit au respect de la vie privée sur la base de l'art. 8 CEDH. ${ }^{70}$ En effet, le droit suisse ne définit pas avec suffisamment de clarté les conditions dans lesquelles le suicide assisté était autorisé. ${ }^{71}$ Cet arrêt qui a été renversé par la Grande Chambre car la requérante avait caché à son avocat ainsi qu'à la Cour son décès avant que la décision fut prise, ${ }^{72}$ a tout de même attiré l'attention du législateur suisse. ${ }^{73}$

Dans l'arrêt mentionné, la Cour EDH relève plus précisé- 20 ment le manque d'orientation quant à la question de savoir si et dans quelles circonstances un médecin est en droit d'établir une ordonnance de $\mathrm{NaP}$ à un patient qui ne souffre pas d'une maladie en phase terminale. ${ }^{74} \mathrm{Ce}$ manque d'orientation juridique claire est susceptible d'avoir un effet dissuasif sur les médecins qui, sinon, seraient disposés à fournir la prescription médicale demandée. ${ }^{75}$ Les autorités suisses ne sont pas moins dispensées de trouver un consensus politique sur la question controversée du droit à mettre fin à ses jours. ${ }^{76}$ Notons toutefois que trois des juges de la Cour ont émis une opinion dissidente sur la question de la clarté des orientations juridiques suisses en matière d'assistance au suicide. ${ }^{77}$ Selon les juges dissidents, la jurisprudence du Tribunal fédéral renvoyant aux directives médicales de l'ASSM définit suffisamment et clairement les circonstances dans lesquelles un médecin est autorisé à établir une ordonnance de NaP. ${ }^{78}$ L'ASSM a depuis adopté une directive étendant le droit à l'assistance médicale au suicide aux patients souhaitant une aide médicale pour mettre fin à leur vie que la mort soit imminente ou non. ${ }^{79}$ Un risque de contrôle négatif plane néanmoins aussi longtemps que la Suisse ne dispose pas d'orientations claires et approuvées par l'État de la portée d'un droit d'obtenir une dose létale de NaP sur prescription médicale. ${ }^{80}$

70 Jugement du Cour EDH n $67810 / 10$ du 14 mai 2013 (Gross c.Suisse), \$69

71 Jugement du Cour EDH n ${ }^{\circ} 67810 / 10$ du 14 mai 2013 (Gross c.Suisse), \$67.

72 Jugement du Grande Chambre du Cour EDH n67810/10 du 30 septembre 2014 (Gross c. Suisse).

73 Pour une étude détaillée voir JEAN-FRANÇOIS PERRIN, Après l'arrêt Gross c. Suisse... Que faut-il faire?, Jusletter 18 août 2014, p. 8.

74 Jugement du Cour EDH n ${ }^{\circ} 67810 / 10$ du 14 mai 2013 (Gross c.Suisse), \$65.

75 Jugement du Cour EDH n67810/10 du 14 mai 2013 (Gross c.Suisse), \$65.

76 Jugement du Cour EDH n ${ }^{\circ} 67810 / 10$ du 14 mai 2013 (Gross c.Suisse), \$66.

77 Voir opinion dissidente commune dans Jugement du Cour EDH $\mathrm{n}^{\circ} 67810 / 10$ du 14 mai 2013 (Gross c. Suisse).

78 Opinion dissidente commune dans Jugement du Cour EDH $\mathrm{n}^{\circ} 67810 / 10$ du 14 mai 2013 (Gross c. Suisse), §1.

79 Directives datées du 17 mai 2018 «Attitude face à la fin de vie et à la mort».

80 Jugement du Cour EDH n ${ }^{\circ} 67810 / 10$ du 14 mai 2013 (Gross c. Suisse), $\S 66,68$ et 69 ainsi que BELSER/EGLI (n. 47), p. 413 ss. 

pays plus libéraux comme les pays du Benelux, le droit suisse gagnerait néanmoins en clarté et contribuerait à la sécurité juridique en fixant dans une loi fédérale la procédure et les conditions visant à obtenir l'assistance médicale au suicide. ${ }^{81}$ Le Tribunal fédéral tente de combler cette lacune dans son arrêt ATF 133 I 58 en consacrant un droit au suicide ainsi qu'en renvoyant aux directives de l'ASSM. ${ }^{82}$ Toutefois, le Tribunal fédéral ne dispose pas en principe de la légitimité démocratique nécessaire pour fixer la procédure et les conditions de la distribution et de l'octroi d'une ordonnance de NaP, tâche incombant en premier lieu à l'Assemblée fédérale. ${ }^{83}$ On ne saurait cependant lui reprocher de combler cette lacune provisoirement, du moment surtout que l'arrêt Gross remonte à bientôt sept ans. Contrairement à l'opinion dissidente commune dans l'arrêt Gross c. Suisse ${ }^{84}$, une telle réforme législative de l'assistance médicale au suicide s'impose non seulement du point de vue des patients souhaitant mettre fin à leur vie, mais permettrait aussi de soulager les professions médicales qui seraient mieux à même d'appréhender les limites du droit à l'autodétermination sans redouter de sanctions administratives ou pénales. ${ }^{85}$

81 Voir notamment BELSER/EGLI (n. 47), p.395 s.

82 ATF 133 I 58 c. 6.3.4.

83 HÜRLIMANN, Kommentar (n. 2), p.1174; TEICHMANN/CAMPRUBI (n. 46), p.30.

84 Pour une position soutenant que les directives de l'ASSM définissent avec suffisamment de clarté les conditions de la distribution de $\mathrm{NaP}$, voir notamment HECTOR ENTENZA, Déterminations sur l'arrêt Gross, RSDIE 2014, p. 352.

85 Voir par exemple FRANK PETERMANN, Die geltende Regelung für Natrium-Pentobarbital : Ein legistischer Rubik's Cube?, AJP 2008, p.1413, GIAN EGE, Die Rechtfertigung der indirekt aktiven Sterbehilfe, dans: Schwarzenegger/Ida (éd.) Autonomie am LebensendeKultur und Recht, p. 114 s., ALINE LÜTHI, Lebensverkürzung im medizinischen Kontext: Behandlungsbegrenzungen und Leidenslinderungen. Ein strafrechtlicher Regelungsvorschlag, thèse Zurich 2014, p. 147.

\section{Conclusion}

Le droit à l'autodétermination soutenu par l'art. 8 CEDH 22 gagne du terrain dans différentes juridictions à l'exemple de la Suisse et du Benelux, bien qu'elles aient adopté des approches différentes quant à l'assistance médicale au suicide. À défaut de prendre position sur un droit au suicide, la Cour EDH renvoie à la marge d'appréciation des états parties à la CEDH quant au droit à l'autodétermination. Le droit suisse relativement libéral tolère quant à lui les organisations d'aide au suicide, mais assujettit l'utilisation de NaP à une autorisation qui elle dépend de conditions médicales, laissant ainsi un vide législatif quant aux personnes désireuses d'euthanasie sans remplir les conditions médicales. En l'état actuel, le droit suisse risque de ne pas résister à un futur examen par la Cour EDH. Dans son ATF 133 I 58, le Tribunal fédéral aura tenté de combler cette lacune en statuant qu'il n'est pas anticonstitutionnel d'exiger une ordonnance médicale pour la distribution de NaP et reconnaissant par la même occasion un droit au suicide. Toutefois, ce vide ne sera définitivement comblé qu'une fois que la Suisse se sera dotée d'une loi au sens formel réglant les conditions et la procédure nécessaire à l'octroi d'une ordonnance médicale pour la distribution de médicaments mortifères, au même titre que le Benelux. 